\title{
CHARACTERISTICS OF RUMINAL MICROBIAL COMMUNITY: EVOLUTIONARY AND ECOLOGICAL PERSPECTIVES
}

\author{
A. K. PATRA ${ }^{1 *}$ \\ ${ }^{1}$ Department of Animal Nutrition, West Bengal University of Animal and Fishery Sciences, \\ Kolkata-700 037, West Bengal, India
}

\begin{abstract}
Ruminants perhaps appeared about 50 million years ago (Ma). Five ruminant families had been extinct and about 200 species in 6 ruminant families are living today. The first ruminant family probably was small omnivore without functional ruminal microbiota to digest fiber. Subsequently, other ruminant families evolved around 18-23 Ma along with woodlands and grasslands. Probably, ruminants started to consume selective and highly nutritious plant leaves and grasses similar to concentrates. By 5-11 Ma, grasslands expanded and some ruminants used more grass in their diets with comparatively low nutritive values and high fibers. Historically, humans have domesticated 9 ruminant species that are mostly utilizer of low quality forages for human benefits. Thus, the non-functional rumen microbiota to predominantly concentrate fermenting microbiota, followed by predominantly fiber digesting microbiota had evolved for mutual complementary benefits of holobiont over the million years. The core microbiome of ruminant species seems the resultant of hologenome interaction in an evolutionary unit. The inertia and resilience properties of ruminal ecosystem seem to be due to this core microbiota, which makes the ecosystem most stable in response to perturbations because this core microbiota has evolutionary advantages with logically more generalists (i.e., wide metabolic versatile and redundancy). Also, a part of the ruminal microbiome shows highly plasticity, which is likely useful for evolutionary adaptability of holobiont. This review discusses ecological characteristics of ruminal microbial community in evolutionary perspectives. The updated understanding of ecological traits of ruminal microbiome would be helpful to better modulate the ruminal fermentation favorably for human benefits.
\end{abstract}

Key words: Ecology, Evolution, Hologenome, Microbiome, Rumen

\section{Introduction}

Humans and ruminants have shared a long history over thousands of years. Prehistoric nomadic hunters perhaps recognized that the photosynthetic potential of grasslands could be exploited by domesticating ruminants to increase their food supply, which would allow them to form stable social communities (Russell and Rychlik, 2001). Humans had, thus, domesticated mostly such ruminant animals that can utilize low-quality fibrous diets (von Engelhardt et al., 1985), not suitable for human consumption and will not compete for their foods. In the last 10,000 years, humans had domesticated nine ruminant species and they naturally prefer some grass in their diets and except reindeer, all belong to Bovidae family (Hackmann and Spain, 2010).

Ruminant animals, ruminal microorganisms, and their biome (holobiome) had co-evolved for millions of years as an evolutionary unit, and the rumen harbors diverse and interdependent populations of bacteria, protozoa including flagellates, archaea, fungi including yeasts, and virus. Ruminants cannot 
produce fiber-degrading enzymes, but bacteria, fungi, and protozoa that are inhabited in the rumen of ruminant animals can digest fibrous materials. The host creates an appropriate habitat for growth of the microbiota, and the microbiota, in turn, supplies short-chain fatty acids (energy source; derived from the fibrous carbohydrates), protein, and vitamins to the animals, which is a complementary mutual approach for a holobiont (host and its microbiota). In the past 60-70 years, humans, however, have significantly changed the foragebased diets of ruminants to grain-based diets (Russell and Rychlik, 2001). Much of the ruminant production system is recently based on grain feeding, especially in technologically developed countries, to improve the productivity of ruminants (Russell and Rychlik, 2001) to fulfill the needs of burgeoning human population, which is against the evolutionary forces. Microorganisms in the rumen can also ferment soluble sugars and starch quickly, and these soluble materials increase fermentation rate. However, ruminants fed on fiber-deficient diets may not have proper homeostatic mechanisms of digesta flow, $\mathrm{pH}$ regulation, and gas removal, and the health and production of ruminants can be adversely affected (Russell and Rychlik, 2001). Furthermore, different nutritional interventions have been explored to modulate ruminal microbiome for enhancing the advantages and minimizing the drawbacks of ruminal fermentation such as reduction of methanogenesis, improvement of fiber digestions, alteration of fatty acid biohydrogenation, and enhancing nitrogen utilization efficiency (Belanche et al., 2020). The modulation of microbiome perhaps has some limitations due to inherent characteristics of the microbiome and hologenome. In this review, different ecological properties of the ruminal microbiota are discussed in an evolutionary viewpoint which will be useful to modulate the ruminal fermentation for obtaining human and animal benefits.

\section{Ruminants in evolutionary perspectives}

The ecology and evolution of ruminants has been reviewed previously (Hackmann and Spain, 2010). Ruminants include any artiodactyl (mammalian in order Artiodactyla) possessing 3-4 chambered stomach (i.e., rumen, reticulum, omasum or isthmus, and abomasum). Tylopods were previously classified as ruminants, but they are not considered in ruminant groups (Cornelis et al., 2013). It has been recognized that 5 ruminant families (i.e., Hypertragulidae, Leptomerycidae, Gelocidae, Palaeomerycidae, and Dromo-merycidae) had been extinct from the world (Hackmann and Spain, 2010). Hypertragulidae, Gelocidae, and Leptomerycidae were small ruminants without horns and they were perhaps most closely similar to moschids or tragulids that are living today (Webb and Taylor, 1980). Dromomerycidae and Palaeomerycidae were medium to large size ruminants with deer-like limb proportions and giraffe-like horns, and their ecological niche was perhaps similar to subtropical deer (Janis and Scott, 1987).

Six ruminant families living today include Tragulidae, Moschidae, Cervidae, Antilocapridae, Giraffidae, and Bovidae (Hackman and Spain, 2010). Tragulidae (chevrotains) are the most primitive among all living ruminant families without changing morphologically to a large extent over evolutionary history, and are small forest-dwelling deer-like animals (Hackman and Spain, 2010). Moschidae (e.g., musk deer) are small forest deer in the Southern Asia, particularly in the Himalayas. The remaining four families, Bovidae (e.g., cattle, sheep, goats, and antelopes), Cervidae (true deer; e.g., red deer, caribou, moose, elk, and whitetailed deer), Giraffidae (giraffe and okapi), and Antilocapridae (pronghorn) are more common (Hackman and Spain, 2010).

The median body weight of modern ruminants is $45 \mathrm{~kg}$ with body weight ranging from about $2 \mathrm{~kg}$ 
(e.g., royal antelope, Salt's dik-dik, and lesser Malay mouse deer) to $800 \mathrm{~kg}$ (e.g., bison, gaur, and Asian water buffalo).

In the evolutionary history of ruminants, Hypertragulidae were possibly the first to be evolved about 50 million years ago in Southeast Asia (Hernández Fernández and Vrba, 2005). This first ruminant family evolved was small $(<5 \mathrm{~kg}$ ) omnivores (Hernández Fernández and Vrba, 2005) without functional rumen microbiota for fiber fermentation and did not ruminate until about 40 million years ago (Jermann et al., 1995). The remaining families appeared approximately 18 to 23 million years ago (Janis, 1982) in Eurasia (Moschidae, Antilocapridae, Dromomerycidae, Palaeomerycidae, Cervidae and Bovidae) and Africa (Giraffidae). In this time, the climate was drier and cool, and consequently temperate woodlands were the most prevalent flora (Janis, 1982; 1993). These ruminant families started to consume primarily leaves or mixture of grass and leaves (Semprebon and Rivals, 2007; DeMiguel et al., 2008). The body weights of these ruminant species were initially about 20 to $40 \mathrm{~kg}$ (Janis, 1982). By around 5 to 11 million years ago, grasslands had expanded (Jacobs et al., 1999), and some ruminant animals continued to include more grass in their diets.

The first domesticated livestock species (ruminants or non-ruminants) was goat at around 8,000 BC in the Near East (Zeder and Hesse, 2000). They were originally domesticated for meat purpose whose population had depleted in the wild due to hunting and predating (Clutton-Brock, 1999; Diamond, 2002). The majority of other eight ruminant species (European and Zebu cattle, Bali cattle, sheep, water buffaloes, reindeer, yaks, and mithuns) were domesticated by 2,500 BC in either the Southern Asia or Near East (Clutton-Brock, 1999). Some of these species, such as the goat, were initially domesticated for meat, but later for milk, transportation, sacrifice, draft, and trade (Clutton-Brock, 1999).

\section{Feeding habits in evolutionary perspectives}

Of approximately 200 ruminant species in six families living today (Hackman and Spain, 2010 ), only about $25 \%$ are "grass and roughage eaters" (e.g., sheep, cattle, water buffalo, and banteng) which are well-equipped with digestive systems to process fibrous forages rich in structural plant cell wall carbohydrates (Hofmann, 1989). More than $40 \%$ of living ruminant species are 'concentrate selectors', which are far less adapted to plant fiber digestion, but are most adapted to processing easily digestible, natural high quality forage diets, rich in soluble cell contents (Hofmann, 1989). Their exceptionally distinct selective ability is based primarily on olfactory signals to choose highly nutritive forage diets. In this category, there are no domesticated ruminant species (i.e., dik-dik, roe deer, and moose). Approximately $35 \%$ of all ruminant species are morphophysiologically intermediate (e.g., goats, sika deer and impala) between the two extreme types and they have a greater extent of forage selectivity and prefer a mixed diet but avoid fiber-rich diets as long and as much as possible (Hofmann, 1989). From the evolutionary trend, concentrate selectors have higher selectivity for plant cell contents, hydrochloric acid producing tissue in abomasum, postruminal (caecocolic) fiber digestion ability, ruminal amylolysis capacity with possibly bypass of solubles (reticular groove), food passage rate, and lower fiber digestion (cellulolysis) capabilities in rumen, total intestinal length, and rumino-reticular weight and capacity relative to body mass, whereas they are vice versa for the grazers (Hofmann, 1989).

\section{Ruminal microbiota}

Ruminal microbial ecosystem is a complex and 
diverse comprising of mostly anaerobic prokaryotes (bacteria and archaea), eukaryotes (protozoa and fungi), and virus. This microbial community has highly correlated microbial interaction networks (Patra et al., 2019) and is essential for coordinated digestion of feeds, especially cell wall carbohydrates. Bacterial community (number $>10^{10} / \mathrm{g}$ of ruminal content) is most diverse and dense with wide ranging metabolic activities. The number of bacterial species present in rumen is not still clear even using next generation sequencing techniques. Kim et al. (2011) predicted 5721 bacterial operational taxonomic unit (OTU) in about 3500 genera and 943 archeal OTU in 670 genera. From metagenomic sequencing of rumen samples of 42 cattle, Stewart et al. (2018) reported 913 novel microbial genomes. However, the same authors recently reported over 4941 microbial genomes after assembling metagenomic sequencing from the rumen of 283 cattle (Stewart et al., 2019). Stewart et al. (2019) compiled 4941 genomes along with other publicly available genome dataset, which resulted in a total of 5845 rumen microbial genomes. The analysis of this total set of genomes indicated the presence of 2690 unique species and 5574 strains of microorganisms and the authors estimated there are about 3276 species in the rumen of cattle. Seshadri et al. (2018) reported 480 bacterial genomes from rumen of different ruminant species in Hungate 1000 collection project. The Hungate collection, as per Stewart et al. (2019), represents only a small part of the diversity in their microbial genomes that have been assembled from metagenomic sequencing. Protozoa (number $10^{5}$ to $10^{6} / \mathrm{g}$ of ruminal content) can contribute up to $50 \%$ of the ruminal microbial biomass due to their larger size and most ruminal protozoa are ciliate species, with a few flagellates (Newbold and Ramos-Morales, 2020). The diversity and density of the protozoa are lower, and they have possibly lower metabolic activity compared with the bacterial community in the rumen. The benefits of protozoa in the rumen are controversial. Protozoa contributes digestion of fiber and stabilization of ruminal $\mathrm{pH}$ by consuming lactate and engulfment of starch, but it is responsible for methane production (endo- and ecto-symbiotic relationship with methanogens) and decreasing the efficiency of protein utilization in ruminants. Rumen fungi $\left(10^{4}\right.$ to $10^{5} / \mathrm{g}$ of ruminal content) may contribute up to $10 \%$ to $20 \%$ of the rumen microbial biomass based on rRNA transcript abundance and chitin measurements (Huws et al., 2018). Rumen fungi are important fiber degraders, especially in poor quality forage-base diets. The close association of ruminal fungi with methanogens has been shown (Edwards et al., 2017), which may increase the activity of fungi and contribute to ruminal methane production (Newbold and Ramos-Morales, 2020). Several genomes and transcriptomes of rumen fungi have been reported recently compared to what was known earlier (Edwards et al., 2017). Yeasts are also present in the rumen (Fernandes et al., 2019), but their roles remain poorly elucidated. Methanogenic archaea inhabit in the gut of both ruminant and non-ruminant animals (Misiukiewicz et al., 2020). Methanogenic archaea ( $10^{8}$ to $10^{9} / \mathrm{g}$ of ruminal content) is responsible for methane production, which is a greenhouse gas. Thus, several strategies have been explored to mitigate methane emissions from ruminants (Patra, 2016). The archaeal diversity is lower than that of the bacterial population and 21 archaeal genomes from rumen have recently been isolated and characterized via the Hungate 1000 (Seshadri et al., 2018). In the majority of the studies, Methanobrevibacter genus is the most abundant methanogens in the rumen, which are hydrogenotrophic producing methane from $\mathrm{H}_{2}$, $\mathrm{CO}_{2}$ and formate produced by bacteria, protozoa, and fungi (Newbold and RamosMorales, 2020). Other important hydrogenotrophic methanogenic genera 
include Methanosphaera, Methanimicrococcus and Methano-bacterium (Newbold and Ramos-Morales, 2020). Methylotrophic methanogens, e.g., Methanosarcinales, Methanosphaera and Methanomassiliicoccaceae, which produce methane from methanol and methylamines, and aceticlastic methanogens, e.g., Methanosarcinales, which generate methane from acetate (Patra et al., 2017; Newbold and Ramos-Morales, 2020) are less abundant in the rumen. The function of rumen virome is poorly known. Recently, genome sequence of ruminal lytic phages has been published (Gilbert et al., 2017), metagenomic analysis of ruminal virome has been performed (Namonyo et al., 2018), and the presence of RNA viruses infecting fungi (mycoviruses) has been reported (Hitch et al., 2019). However, their effects on ruminal fermentation, microbiota and feed degradation require further investigation. The ruminal microbiome possesses a few distinct ecological characteristics, which have been discussed below including evolutionary perspectives.

\section{Host individuality}

Recently, host microbiome individuality concept has received considerable interest, which means gut microbiota contains a set of taxa common in all animals (a "core microbiome"). There are also a large number of taxa, which varies among hosts. The hologenome (microbiome plus host genome) theory suggests that the microbiome and their genome jointly make an evolutionary unit undergoing adaptation in the selection pressure (Shapira, 2016). The microbiota comprises a multilayered community structures, i.e., a host-adapted core microbial pool, an environmentally modulated flexible microbial community, and a range of intermediates in between them (Shapira, 2016).

The rumen of cattle has been shown to contain a core microbiome in different studies (Jami and
Mizrahi, 2012; Petri et al., 2013). Sasson et al. (2017) indicated that a number of ruminal bacterial operational taxonomic units (OTU, a proxy for species) were greatly heritable in dairy cattle. In a recent study, lambs shifted from a non-grazing concentrate diet to a forage grazing diet had a core microbiota composed of 34 for bacterial genera, which were shared by all sheep, regardless of diets (Belanche et al., 2019). The core bacterial community was formed by Prevotella, Treponema, Ruminobacter, Succinivibrio, Fibrobacter, Sporobacter, Ruminococcus, Anaeroplasma and Selenomonas, representing $20 \%$ of the ruminal bacterial community composition. Moreover, this core community composition was mostly similar to those reported in another study with dairy cattle fed different diets in which 32 genera were common to 16 animals (Jami and Mizrahi, 2012). By analyzing microbial community in 742 samples of rumen content of 32 animal species from 35 countries, seven abundant bacterial groups, Prevotella, Ruminococcus, and Butyrivibrio as well as unclassified Bacteroidales, Clostridiale, Lachnospiraceae and Ruminococcaceae, comprising of $67.1 \%$ of all bacterial sequences were detected as a "core bacterial microbiome" (Henderson et al., 2015). Stewart et al. (2019) suggested that Proteobacteria would also fall into the core rumen microbiome along with above 7 bacterial groups. However, abundances of these core bacterial groups were not equal among all animal species (Henderson et al., 2015). Across different studies, the core ruminal microbiota of cattle generally includes members of Firmicutes family (particularly Ruminococcus and Butyrivibrio genera) and Bacteroidetes family (especially Prevotella genus), together with a number of taxa present in lower abundance. The core microbiota composition is stable independent of diet or host genetic differences (Petri et al., 2013; Henderson et al., 2015).

The host specificity of the ruminal microbiota 
is not only restricted to bacterial domain, because it has also been reported for the members of Archaeal and protozoal communities (Zhou et al., 2012). In beef cattle, relative abundances of archaea were consistent indicating that archaeal abundance in the rumen is under host genetic influence (Roehe et al., 2016). In sheep, 9 and 13 genera of methanogens and fungi are comprised of the core microbiota (Belanche et al., 2019). It has been suggested that as number of animals in each study was small, the number of genera in the core microbiome may be lowered with the inclusion of a global population of hosts (Weimer, 2015). The metabolic functions to the members of this core community are broad performing as 'generalists'.

Much research has now centered on the elucidation of core microbiome in the rumen, which may be a major regulator of overall

A

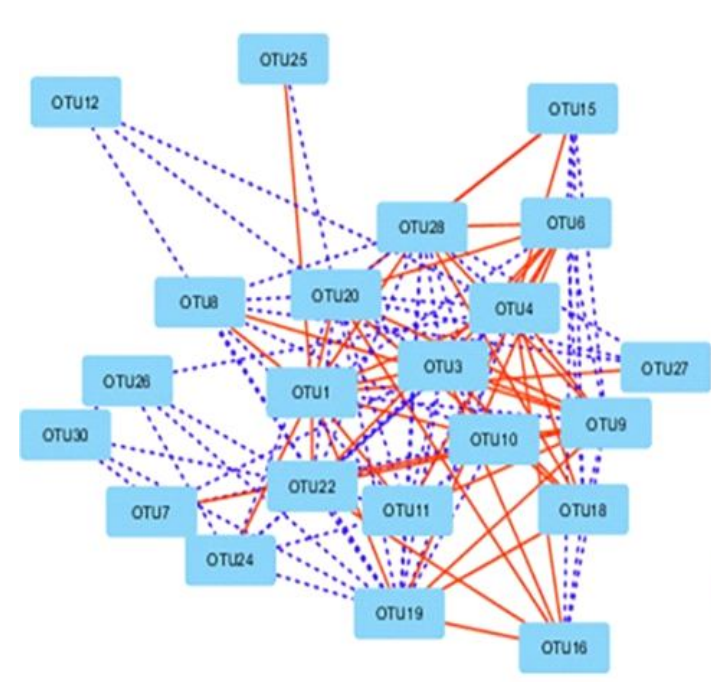

ruminal fermentation stabilizing other microorganisms in the ruminal ecosystem. Because few ruminal microbial species are highly correlated with other species (Fig. 1; Patra et al., 2019), the identification of this species in the core microbiome, if present, would be useful for understanding better rumen function. Also, because this core microbiome is a part of holobiont, correlations between host genome and bacterial genome may identify the drivers of this core microbiome. At this point of research, it is unclear when this compositionally and functionally unique community is assembled, what biotic and abiotic factors determine an early establishment and maintenance of this community, and whether any of its core members have a large influence on the ruminal environment (Weimer, 2015). The use of recent metagenomic and bioinformatic tools may unravel this scientific understanding of the poorly characterized core ruminal microbiome in future.

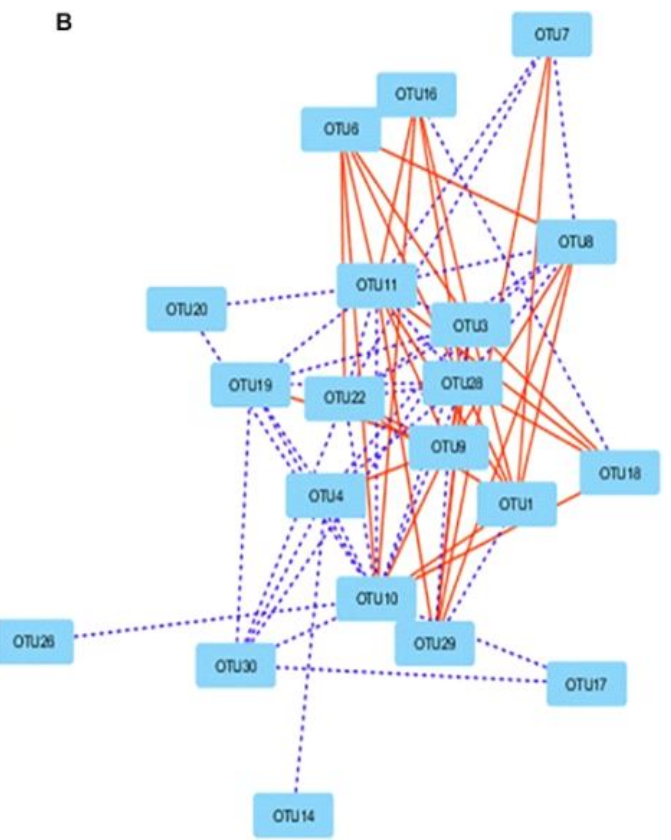

Fig. 1. Correlation networks showing correlations between major operational taxonomic unit (OTUs, a proxy for species; each representing abundance of $\geq 0.5 \%$ total sequence abundances) in the rumen of the control sheep (A) and menthol-fed sheep (B). Dotted lines indicate positive correlations, while solid lines represent negative correlations. The shorter the distance between two nodes, the stronger the correlation between them (Patra et al., 2019). 


\section{Redundancy}

In a microbial community, redundancy is the overlapping metabolic and physiological functions of multiple microbial taxa. This means a number of microbial specifies can degrade a particular feed substrate (e.g., different polymeric substrates, monomeric units, and linkages between monomeric units) and utilize intermediate metabolites. Although there is great complexity and diversity of feeds consumed by ruminants, the small number of degradation sites of feeds or monomeric units and metabolites is relatively less (Weimer, 2015). Conversely, there are a large number of microbial species in the rumen. For instance, most next generation sequence studies have obtained 100-1000 of OTU in ruminal samples (Weimer, 2015). This indicates that there are usually several species that potentially degrade each substrate and metabolize each intermediate (Fig. 2). Moreover, several species can contribute to the degradation of a number of substrates or breakdown many linkages in feeds as 'generalists'. For example, xylan or xyloglucan can be degraded or pyruvate can be metabolized to acetate by the eight major genera representing $70 \%$ of the total abundances (Fig. 2) (Seshadri et al., 2018). Again, Fibrobacter can participate in the many points in the breakdown and metabolism of

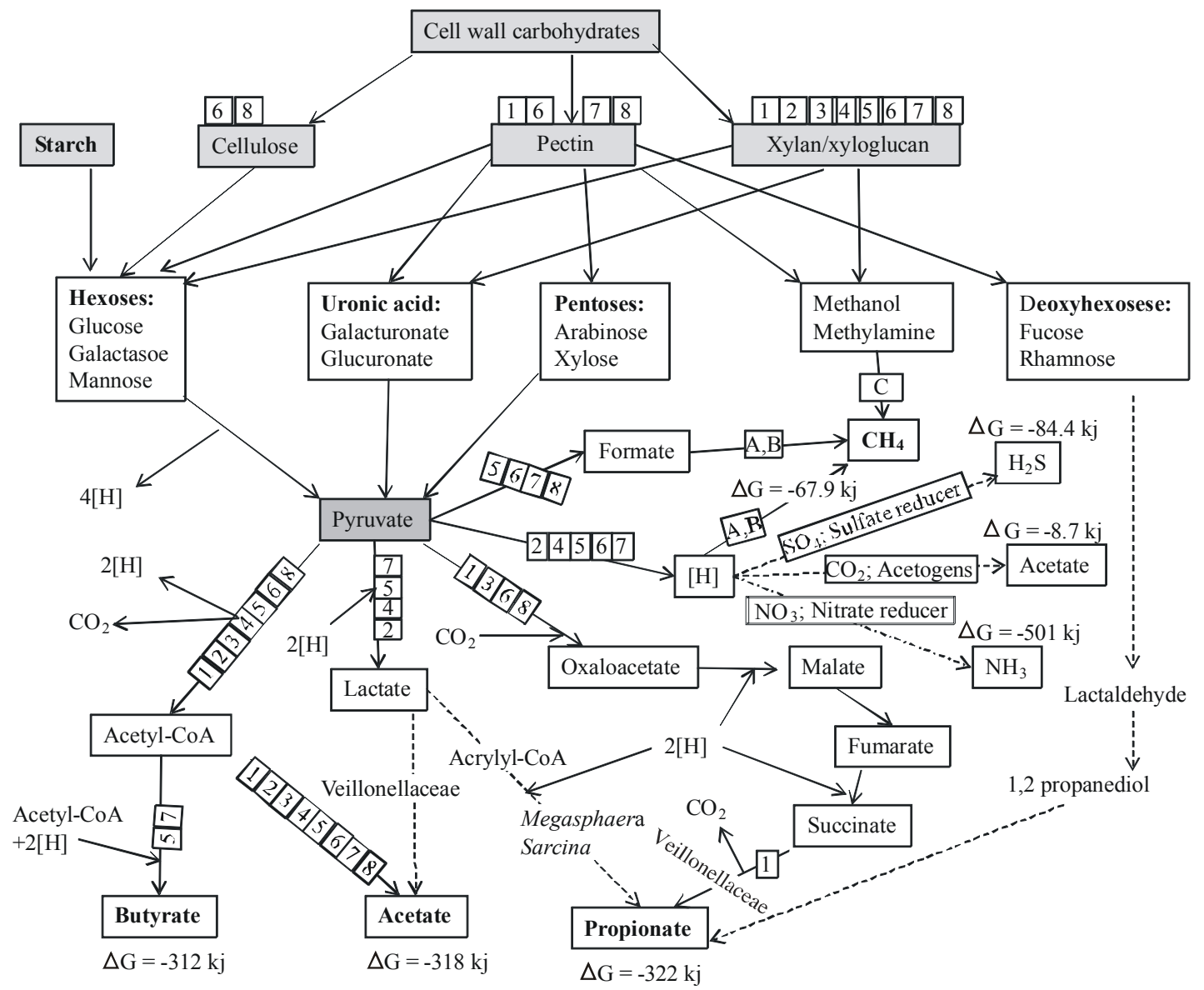

Fig. 2. Fermentation of carbohydrate and their products by ruminal microbiota. 1- Prevotella, 2 - Clostridiales, 3- Bacteroidales, 4 - Ruminococcaceae, 5 - Lachnospiraceae, 6 - Ruminococcus, 7 - Butyrivibrio, 8 Fibrobacter. A - Methanobrevibcter gottschalkï, B - Methanobacter ruminantium, C-Methanomassiliicoccales group $12 \mathrm{sp}$. Solid lines indicate the fermentation by dominant pathways and the dotted lines indicate the other minor pathways. Adapted with modifications (Seshadri et al., 2018; Beauchemin et al., 2020). 
carbohydrate (Fig. 2). Besides carbohydrate degradation, Prevotella and Butyrivibrio, two most abundant bacterial genera with numerous OTU from each of these genera (Pitta et al., 2010; Li et al., 2012; Mohammed et al., 2014) display extremely a wide range of degradative capabilities including proteins and peptide hydrolysis, and fermentation of several amino acids, and intermediates (Kelly et al., 2010). Therefore, it is evident that the microbial community in the rumen is highly redundant in its composition from a metabolic aspect. However, it is unclear which particular species or strain performs a degradative function predominantly in situ when many competitors and symbionts are present in the degradation sites (Weimer, 2015). For instance, Butyrivibrio fibrisolvens YE44 showed rapid and dense growth in the presence of wheat starch as the sole energy substrate and carbon source in the in vitro growth medium, this capability was not evident in the in vivo conditions upon introduction to the rumen of cattle fed grain rich diets because its abundance declined rapidly and was not detectable eight days after introduction (Klieve et al., 2003). Perhaps the role of Butyrivibrio as a starch degrader is minimal as other starch degraders outcompete Butyrivibrio in vivo. Acetogens that produce acetate using hydrogen and carbon dioxide are present in the rumen at a low number, but no acetate is produced from carbon dioxide in the rumen confirmed using labeling studies (LeVan et al., 1998). The acetogens is not thermodynamically favorable to compete with the methanogens for available hydrogen, as indicated by their poorer affinity for hydrogen, lower free energy yield per mole of hydrogen consumed, and slower growth rates (Ungerfeld, 2013). Still acetogens can present in the rumen in low number due to their versatile capability to survive by catabolizing a broad range of organic compounds (Rieu-Lesme et al., 1996). It has been suggested that acetogens in the rumen may participate in reduction of carbon dioxide with hydrogen to produce acetate when the competitors such as methanogens are inhibited. In general, despite the presence of keystone functional groups of microbiota, such as fibrolytic microbes and methanogens, many species represent each of these functional groups in the rumen. Due to this redundancy, it is doubtful that any one species would have a special role in the rumen contributing predominantly to the ruminal fermentation, which would be designated as keystone species (Weimer, 2015).

It has also been predicted that catabolic redundancy of the ruminal microbiota would be greater for the abundant and degradable substrates and their monomers (Weimer, 2015). Consequently, the number of species metabolizing glucose becomes higher than those can degrade less abundant substrates; for example, oxalate (Weimer, 2015). Thus, the ruminal microbial community contains a mix of 'generalists' that utilize many abundant substrates, and 'specialists' that utilize a smaller number of less abundant substrates and also face much less competition. Therefore, despite substantial changes in microbial community composition, alterations in fermentation characteristics such as $\mathrm{pH}$, volatile fatty acid concentrations or molar proportions are not reflected as a result of redundancy of the ruminal microbial community (Sandri et al., 2014; Patra et al., 2019). A study with dietary menthol in lambs showed that menthol altered a few microbiota compositions, including a decrease in certain methanogens numbers, an increase in microbiota diversity, and microbial and metabolic networks (Patra et al., 2019). However, these changes did not translate to ruminal microbial fermentation because the ruminal species interacted both positively and negatively in response to menthol (Fig. 1), indicating that stable ruminal fermentation is maintained in spite of minor microbial changes due to metabolic redundancy of the ruminal ecosystem (Patra et al., 2019). 


\section{Resilience}

Ruminal microbiota responds to perturbation by different physical (e.g., temperature), chemical (e.g., diet composition, ruminal $\mathrm{pH}$ or plant toxins), or biological (e.g., introduction of non-native microbes) changes like any microbial ecosystem (Weimer, 2015). Intensity, frequency and duration of perturbations may differ, which may determine the nature of the microbial responses. Some perturbations, for example, variations in feed intake patterns or water consumption are common features, whereas other perturbations such as gradual change in forage quality in grazing fields are noticeable in the ruminal environment (Weimer, 2015). The response of the ruminal microbiome to these perturbations can be better explained in terms of inertia and its resilience (Westman, 1978; Weimer, 2015). From classical ecology theory (Westman, 1978), inertia (i.e., ability to resists a change) reflects the stability of the ruminal ecology, while resilience indicates how the ruminal microbial community returns back once its community has been altered. In ecosystem, the properties of inertia and resilience are simply examined at the individual species level over time or across spatial domains (Westman, 1978). Because of high species diversity and complex interactions among organisms in ruminal habitats, exploring the influencing factors of inertia and resilience would be difficult for individual species and more difficult for entire microbial communities (Weimer, 2015). Nevertheless, these concepts could be useful to theoretically supporting the discussions on how successfully the ruminal microbiota and fermentation might be manipulated (Weimer, 2015).

Few experiments were performed for dosing with fibrolytic microbes in the rumen to improve fiber digestibility. But inoculation of fiber degrading microbes of ruminal origin has not been of much success due to resilience nature of the ruminal communities (Weimer, 2015). Varel et al. (1995) isolated a Clostridium longisporum strain from the rumen of bison, and a Clostridium herbivorans strain from the intestinal tract of pigs, which had greater fiber degrading activity in pure culture than the common ruminal cellulolytic bacteria (Fibrobacter succinogenes, Ruminococcus albus, and Ruminococcus flavefaciens). These fermenter-grown Clostridium cultures were dosed into ruminal cannulated cows whose rumen content was almost completely emptied and then the cows were fed with hay (Varel et al., 1995). These Clostridium strains were expected to establish in the rumen of cows fed fiber rich hay diets. However, these strains were not detectable, generally within $24 \mathrm{~h}$ of dosing. These strains were isolated from the rumen or hindgut, but they are present at low levels in the ruminal habitat, which indicated that these Clostridium strains were not very much competitive in the rumen and the quantitatively modest residual ruminal community was quickly and easily capable to expel the inoculated species. It may suggest that native (autochthonous) fiber hydrolyzing microbes in the rumen of each individual animal are adequately adapted in their native habitat to oust non-native (allochthonous) species from other habitats, including the rumen of other animals (Weimer, 2015). Besides abiotic stressors, an introduced species or strain needs to face many opponents, i.e., direct competitors along with their co-adapted symbionts with which they have productive mutualistic relationships, and finds few friendly microbes that may get immediate benefits from cooperating with the dosed species (Weimer, 2015).

In contrast to cellulolytic microbes that have many competitors, establishment of dosed strains with non-fibrolytic 'specialist' microbes in the rumen has been successful in a few cases. For example, ruminal inoculation of Synergistes jonesii to degrade minosine present in Leucaena leucocephala shrub conferred resistance to mimosine poisoning in the recipient animals (Jones and Megarrity, 1986; Allison et al., 1992; Hammond, 1995). In another study, Megasphaera elsdenii YE34 (a lactate utilizer) culture isolated from the rumen of cattle was introduced to cattle, which immediately established in the rumen of 
inoculated grain fed cattle, 7-10 days earlier than in uninoculated cattle (Klieve et al., 2003). Similarly, dosing of a recombinant strain of ruminal Butyrivibrio fibrisolvens containing dehalogenase gene from a Pseudomonas strain successfully established in sheep for several months and provided enhanced tolerance to fluoroacetate (Gregg et al., 1998). These studies suggested that inoculation success depends upon the introduced species or strains that fill an open niche along with highly specialized metabolic activities and substantial substrate concentrations. There are also a few other specialists such as nitrate reducers and sulfate reducers which can act as electron acceptors in the rumen and they are metabolically favorable compared with the methanogens (Fig. 2). However, the substrate availability for these 'specialists' that have less competitors are low in the rumen. Increasing the substrate availability may increase their abundances and reduce methane production in the rumen (Patra et al., 2017).

\section{Plasticity}

Outside the core microbiome, there is perhaps extensive variability and metabolic plasticity of the microbiota composition in the rumen among individual animals, which is altered easily in response to diets, genetics and other abiotic factors. Microbial diversity and density change in response to diets (Petri et al., 2013; Henderson et al., 2015). For an example, when sheep were shifted from a concentrate-hay diet to pasture diet, diet-specific core microbial community was small in size $(<2 \%)$ but had increased concentrations and diversity, which suggested that degradation of pasture is a complex process which needs a coordinated work by the diverse consortium of microbiota (Belanche et al., 2019). The correlations between the ruminal fermentation variables and abundance of microbial taxa were not consistent across diets, which suggest that microbiota adapted to different substrates and shifted their fermentation products (Belanche et al., 2019). Also, both the overall structure of the methanogenic community and the abundance of $55 \%$ of the methanogens taxa were affected by the diets consumed by the ruminant indicating a larger diet adaptation process for the methanogens than for the bacterial community (Popova et al., 2013). This may suggest that most of the adaptation processes to the diets do not replace bacterial genera but increase abundance or diversity of microbiota or modified their metabolic activity (Belanche et al., 2019). Moreover, microbiome composition after feeding changes considerably manifested as substantial alterations in the relative abundance (3-5-folds) of methanogens and major bacterial taxa such as Prevotella, which was independent of individual host variation and diet (Shaani et al., 2018). The high plasticity of the ruminal microbial community in response to dietary and ruminal environmental pressure is likely to be useful for evolutionary adaptability of the holobiont.

\section{Co-occurrence}

The presence of a microbe in the rumen is highly dependent on other microbes. The classical example is ecto- and endo-symbiotic relations between protozoa and methanogens (Patra et al., 2017). Therefore, defaunation (reduction of protozoal number) causes reduced number of total methanogens in the rumen, at least, some methanogenic species (Patra et al., 2016; 2017). Ruminal fungi and methanoogens may also have a symbiotic relation (Edwards et al., 2017) where both communities seem share mutual complementary metabolic activities. Similarly, the correlated networks of the bacterial species may suggest cooccurrences and interdependence of some bacterial species, but this relation is not well studied (Patra et al., 2019).

\section{Conclusions}

Ruminal microbial community has co-evolved as a part of hologenome of an evolutionary unit over 20-30 millions years. Thus, the core microbiota of a ruminant species seems the resultant of the host-microbiota (symbiont) interaction for mutual benefits. The inertia and resilience properties of ruminal ecosystem seems to be due to this core microbiota, which make the ecosystem most stable in response to biotic and abiotic forces in the rumen because 
this core microbiota has evolutionary advantages. This core microbiota has logically more generalists with wide metabolic versatile and redundancy. Also, a part of the ruminal microbial community shows highly plasticity in response to dietary and ruminal environmental pressure, which

\section{REFERENCES}

Allison MJ, Mayberry WR, McSweeney CS and Stahl DA, 1992. Synergistes jonesii, gen. nov., sp. nov: A rumen bacterium that degrades toxic pyridinediols. Syst Appl Microbiol, 15(4): 522529, doi: 10.1016/S0723-2020(11) 80111-6

Beauchemin KA, Ungerfeld EM, Eckard RJ and Wang M, 2020. Review: fifty years of research on rumen methanogenesis: lessons learned and future challenges for mitigation. Animal, 14(S1): S2S16, doi: 10.1017/S1751731119003100

Belanche A, Kingston-Smith AH, Griffith GW and Newbold CJ, 2019. A multi-kingdom study reveals the plasticity of the rumen microbiota in response to a shift from non-grazing to grazing diets in sheep. Front Microbiol, 10: 122, doi: 10.3389/fmicb.2019.00122

Belanche A, Patra AK, Morgavi DP, Suen G, Newbold CJ et al., 2020. Editorial: Gut microbiome modulation in ruminants: enhancing advantages and minimizing drawbacks. Front Microbiol, doi: 10.3389/fmicb.2020.622002

Clutton-Brock J, 1999. A Natural History of Domesticated Mammals. $2^{\text {nd }}$ edn. Cambridge Univ Press, Cambridge, UK

Cornelis G, Heidmann O, Degrelle SA, Vernochet C, Lavialle C et al., 2013. Captured retroviral envelope syncytin gene associated with the unique placental structure of higher ruminants. Proc Natl Acad Sci, U S A, 110: E828-E837, doi: 10.1073/pnas. 1215787110

DeMiguel D, Fortelius M, Azanza B and Morales J, 2008. Ancestral feeding state of ruminants reconsidered: earliest grazing adaptation claims a mixed condition for Cervidae. BMC Evol Biol, 8: 13, doi: 10.1186/1471-2148-8-13

Diamond J, 2002. Evolution, consequences and future of plant and animal domestication. seems to be useful for evolutionary adaptability of the holobiont. The updated understanding of host-specificity, redundancy, resilience and plasticity of the ruminal microbiome ecology would be useful to better modulate the ruminal fermentation in a favorable way and obtain benefits from ruminants.

Nature, 418(6898): 700-707, doi: 10.1038/ nature01019.

Edwards JE, Forster RJ, Callaghan TM, Dollhofer V, Dagar SS et al., 2017. PCR and omics based techniques to study the diversity, ecology and biology of anaerobic fungi: insights, challenges and opportunities. Front Microbiol, 8: 1657, doi: 10.3389/fmicb.2017.01657

von Engelhardt W, Dellow DW and Hoeller H, 1985. The potential of ruminants for the utilization of fibrous low-quality diets. Proc Nutr Soc, 44(1): 37-43, doi: 10.1079/pns19850008

Fernandes T, Carvalho BF, Mantovani HC, Schwan RF and Ávila CLS, 2019. Identification and characterization of yeasts from bovine rumen for potential use as probiotics. J Appl Microbiol, 127(3): 845-855, doi: 10.1111/jam. 14350

Gilbert RA, Kelly WJ, Altermann E, Leahy SC, Minchin, C et al., 2017. Toward understanding phage: host interactions in the rumen; complete genome sequences of lytic phages infecting rumen bacteria. Front Microbiol, 8: 2340, doi: 10.3389/fmicb.2017.02340

Gregg K, Hamdorf B, Henderson K, Kopecny J and Wong C, 1998. Genetically modified ruminal bacteria protect sheep from fluoroacetate poisoning. Appl Environ Microbiol, 64(9): 3496-3498, doi: 10.1128/AEM.64.9.34963498.1998

Hackmann TJ and Spain JN, 2010. Invited review: Ruminant ecology and evolution: perspectives useful to ruminant livestock research and production. J Dairy Sci, 93(4): 13201334, doi: $10.3168 /$ jds.2009-2071

Hammond AC, 1995. Leucaena toxicosis in ruminants and its control. J Anim Sci, 73(5): 1487-1492, doi: $10.2527 / 1995.7351487 x$ 
Henderson G, Cox F, Ganesh S, Jonker A, Young W et al., 2015. Rumen microbial community composition varies with diet and host, but a core microbiome is found across a wide geographical range. Sci Rep, 5: 14567, doi: 10.1038/srep14567

Hernández Fernández M and Vrba ES, 2005. A complete estimate of the phylogenetic relationships in Ruminantia: A dated species-level supertree of the extant ruminants. Biol Rev Camb Philos Soc, 80(2): 269-302, doi: 10.1017/ s 1464793104006670

Hitch TCA, Edwards JE and Gilbert RA, 2019. Metatranscriptomics reveals mycoviral populations in the ovine rumen. FEMS Microbiol Lett, 366(13): fnz161, doi: 10.1093/femsle/ fnz161

Hofmann RR, 1989. Evolutionary steps of ecophysiological adaptation and diversification of ruminants: A comparative view of their digestive system. Oecologia, 78(4): 443-457, doi: 10.1007/BF00378733

Huws SA, Creevey CJ, Oyama LB, Mizrahi I, Denman SE et al., 2018. Addressing global ruminant agricultural challenges through understanding the rumen microbiome: past, present, and future. Front Microbiol, 9: 2161, doi: 10.3389/ fmicb.2018.02161

Jacobs BF, Kingston JD and Jacobs LL, 1999. The origin of grass-dominated ecosystems. Ann Mol Bot Gard, 86(2): 590-643, doi: 10.2307/2666186

Jami E and Mizrahi I, 2012. Composition and similarity of bovine rumen microbiota across individual animals. PLoS One, 7(3): e33306, doi: 10.1371/ journal.pone. 0033306

Janis C, 1982. Evolution of horns in ungulates: ecology and paleoecology. Biol Rev, 57(2): 261-318, doi: 10.1111/j.1469-185X.1982.tb00370.x

Janis CM, 1993. Tertiary mammal evolution in the context of changing climates, vegetation, and tectonic events. Annu Rev Ecol Syst, 24: 467500, doi: 10.1146/annurev.es.24.110193.002343

Janis CM and Scott KM, 1987. The interrelationships of higher ruminant families with special emphasis on the members of the Cervoidea. Am Mus Novit, 2893: 1-85
Jermann TM, Opitz JG, Stackhouse J and Benner SA, 1995. Reconstructing the evolutionary history of the artiodactyl ribonuclease superfamily. Nature, 374(6517): 57-59, doi: 10.1038/ $374057 \mathrm{a} 0$

Jones RJ and Megarrity RG, 1986. Successful transfer of DHP-degrading bacteria from Hawaiian goats to Australian ruminants to overcome the toxicity of Leucaena. Aust Vet J, 63(8): 259-262, doi: 10.1111/j.1751-0813.1986.tb02990.x

Kelly WJ, Leahy SC, Altermann E, Yeoman CJ, Dunne JC et al., 2010. The glycobiome of the rumen bacterium Butyrivibrio proteoclasticum B316(T) highlights adaptation to a polysaccharide-rich environment. PLoS One, 5(8): e11942, doi: 10.1371/journal.pone.0011942

Kim M, Morrison M and Yu Z, 2011. Status of the phylogenetic diversity census of ruminal microbiomes. FEMS Microbiol Ecol, 76(1): 4963, doi: 10.1111/j.1574-6941. 2010.01029.x

Klieve AV, Hennessy D, Ouwerkerk D, Forster RJ, Mackie RI et al., 2003. Establishing populations of Megasphaera elsdenii YE 34 and Butyrivibrio fibrisolvens YE 44 in the rumen of cattle fed high grain diets. J Appl Microbiol, 95(3): 621-30, doi: 10.1046/j.1365-2672.2003.02024.x

LeVan TD, Robinson JA, Ralph J, Greening RC, Smolenski WJ et al., 1998. Assessment of reductive acetogenesis with indigenous ruminal bacterium populations and Acetitomaculum ruminis. Appl Environ Microbiol, 64(9): 34293436, doi: 10.1128/AEM.64.9.3429-3436.1998

Li RW, Connor EE, Li C, Baldwin VRL and Sparks ME, 2012. Characterization of the rumen microbiota of pre-ruminant calves using metagenomic tools. Environ Microbiol, 14(1): 129-139, doi: 10.1111/j.1462-2920.2011.02543.x

Misiukiewicz A, Gao M, Filipiak W, Cieslak A, Patra AK et al., 2020. Review: Methanogens and methane production in the digestive systems of non-ruminant farm animals. Animal, doi: 10.1016/j.animal.2020.100060

Mohammed R, Brink GE, Stevenson DM, Neumann AP, Beauchemin KA et al., 2014. Bacterial communities in the rumen of Holstein heifers differ when fed orchardgrass as pasture vs. 
hay. Front Microbiol, 5: 689, doi: 10.3389/ fmicb.2014.00689

Namonyo S, Wagacha M, Maina S, Wambua L and Agaba M, 2018. A metagenomic study of the rumen virome in domestic caprids. Arch Virol, 163(12): 3415-3419, doi: 10.1007/ s00705-018-4022-4

Newbold CJ and Ramos-Morales E, 2020. Review: Ruminal microbiome and microbial metabolome: Effects of diet and ruminant host. Animal, 14(S1): S78-S86, doi: 10.1017/S1751731119003252

Patra A, Park T, Kim M and Yu Z, 2017. Rumen methanogens and mitigation of methane emission by anti-methanogenic compounds and substances. J Anim Sci Biotechnol, 8: 13, doi: 10.1186/s40104-017-0145-9

Patra AK, 2016. Recent advances in measurement and dietary mitigation of enteric methane emissions in ruminants. Front Vet Sci, 3: 39, doi: 10.3389/ fvets.2016.00039

Patra AK, Park T, Braun HS, Geiger S, Pieper R et al., 2019. Dietary bioactive lipid compounds rich in menthol alter interactions among members of ruminal microbiota in sheep. Front Microbiol, 10:2038, doi: 10.3389/fmicb. 2019.02038

Petri RM, Schwaiger T, Penner GB, Beauchemin KA, Forster RJ et al., 2013. Characterization of the core rumen microbiome in cattle during transition from forage to concentrate as well as during and after an acidotic challenge. PLoS One, 8(12): e83424, doi: 10.1371/journal.pone.0083424

Pitta DW, Pinchak E, Dowd SE, Osterstock J, Gontcharova V et al., 2010. Rumen bacterial diversity dynamics associated with changing from bermudagrass hay to grazed winter wheat diets. Microb Ecol, 59(3): 511-522, doi: 10.1007/ s00248-009-9609-6

Popova M, Morgavi DP and Martin C, 2013. Methanogens and methanogenesis in the rumens and ceca of lambs fed two different high-graincontent diets. Appl Environ Microbiol, 79: 17771786, doi: 10.1128/AEM.03115-12

Rieu-Lesme F, Morvan B, Collins MD, Fonty G and Willems A, 1996. A new H2 /CO2-using acetogenic bacterium from the rumen: description of Ruminococcus schinkii sp. nov. FEMS Microbiol Lett, 140(2-3): 281-286, doi: 10.1016/ 0378-1097(96)00195-4

Roehe R, Dewhurst RJ, Duthie CA, Rooke JA, McKain N et al., 2016. Bovine host genetic variation influences rumen microbial methane production with best selection criterion for low methane emitting and efficiently feed converting hosts based on metagenomic gene abundance. PLoS Genet, 12(2): e1005846, doi: 10.1371/journal.pgen.1005846

Russell JB and Rychlik JL, 2001. Factors that alter rumen microbial ecology. Science, 292(5519): 11191122, doi: $10.1126 /$ science. 1058830

Sandri M, Manfrin C, Pallavicini A and Stefanon B, 2014. Microbial diversity of the liquid fraction of rumen content from lactating cows. Animal, 8(4): 572-579, doi: 10.1017/ S1751731114000056

Sasson G, Ben-Shabat SK, Seroussi E, DoronFaigenboim A, Shterzer N et al., 2017. Heritable bovine rumen bacteria are phylogenetically related and correlated with the cow's capacity to harvest energy from its feed. MBio, 8(8): e0070317, doi: 10.1128/mBio.00703-17

Semprebon GM and Rivals F, 2007. Was grass more prevalent in the pronghorn past? An assessment of the dietary adaptations of Miocene to recent Antilocapridae (Mammalia: Artiodactyla). Palaeogeogr Palaeoclimatol Palaeoecol, 253(34): 332-347, doi: 10.1016/j.palaeo.2007.06.006

Seshadri R, Leahy SC, Attwood GT, Teh KH, Lambie SC et al., 2018. Cultivation and sequencing of rumen microbiome members from the Hungate 1000 collection. Nat Biotechnol, 36(4): 359-366, doi: $10.1038 /$ nbt.4110

Shaani Y, Zehavi T, Eyal S, Miron J and Mizrahi I, 2018. Microbiome niche modification drives diurnal rumen community assembly, overpowering individual variability and diet effects. ISME J, 12(10): 2446-2457, doi: 10.1038/s41396-018-0203-0

Shapira M, 2016. Gut microbiotas and host evolution: scaling up symbiosis. Trends Ecol Evol, 31(7): 539-549, doi: 10.1016/j.tree.2016.03.006 
Stewart RD, Auffret MD, Warr A, Walker AW, Roehe $\mathrm{R}$ et al., 2019. Compendium of 4,941 rumen metagenome-assembled genomes for rumen microbiome biology and enzyme discovery. Nat Biotechnol, 37: 953-961, doi: 10.1038/s41587019-0202-3

Stewart RD, Auffret MD, Warr A, Wiser AH, Press MO et al., 2018. Assembly of 913 microbial genomes from metagenomic sequencing of the cow rumen. Nature Commun, 9(1): 870, doi: 10.1038/s41467018- 03317-6

Ungerfeld EM, 2013. A theoretical comparison between two ruminal electron sinks. Front Microbiol, 4: 319, doi: 10.3389/fmicb.2013.00319

Varel VH, Yen JT and Kreikemeier KK, 1995. Addition of cellulolytic clostridia to the bovine rumen and pig intestinal tract. Appl Environ Microbiol, 61(3): 1116-1119

Webb SD and Taylor BE, 1980. The phylogeny of hornless ruminants and a description of the cranium of Archaeomeryx. Bull Am Mus Nat Hist, 167: 120-157

Weimer PJ, 2015. Redundancy, resilience, and host specificity of the ruminal microbiota: implications for engineering improved ruminal fermentations. Front Microbiol, 6: 296, doi: 10.3389/fmicb. 2015.00296

Westman WE, 1978. Measuring the inertia and resilience of ecosystems. BioSci, 28(11): 705-710, doi: $10.2307 / 1307321$

Zeder MA and Hesse B, 2000. The initial domestication of goats (Capra hircus) in the Zagros mountains 10,000 years ago. Science, 287(5461): 22542257, doi: 10.1126/science.287.5461.2254

Zhou M, Hünerberg M, Beauchemin KA, McAllister TA, Okine EK et al., 2012. Individuality of ruminal methanogen/protozoan populations in beef cattle fed diets containing dried distillers' grain with solubles. Acta Agric Scand A Anim Sci, 62(4): 273288, doi: 10.1080/09064702.2013.788206 